\title{
Cognitive impairments of alcoholic cirrhotic patients: correlation with endogenous benzodiazepine receptor ligands and increased affinity of platelet receptors
}

Flavio Kapczinski, H Valerie Curran, Robert Przemioslo, Roger Williams, Emma Fluck, Cathy Fernandes, Sandra E File

\begin{abstract}
Objectives-To determine whether differences in cognitive function between alcoholic and non-alcoholic cirrhotic patients relate to differences in endogenous ligands for the benzodiazepine receptor and/or benzodiazepine binding.

Methods-Seventeen grade-I hepatic encephalopathic patients (nine alcoholic, eight non-alcoholic) were compared with 10 matched controls on plasma concentrations of endogenous ligands for the neuronal benzodiazepine receptor, benzodiazepine binding in platelets, and performance on tests of cognitive function. Results-Both groups of patients were impaired on verbal recall and on reaction time tasks compared with controls; alcoholic patients were also impaired on Reitan's trails test and digit cancellation. Four of the 17 patients had detectable concentrations of endogenous benzodiazepine ligands and they were more impaired than other patients on trails and cancellation tests. The groups did not differ in the density of benzodiazepine platelet receptors, but receptor affinity was higher in alcoholic patients than in controls; furthermore, receptor affinity correlated with the time to complete the cancellation task and with reaction time. Conclusion-Alcoholic cirrhotic patients may have enhanced concentrations of ligands for neuronal and peripheral benzodiazepine receptors and these may contribute to cognitive impairments in these patients.
\end{abstract}

$(\mathcal{F}$ Neurol Neurosurg Psychiatry 1996;60:676-680)

Keywords: benzodiazepines; alcoholic patients; cirrhosis; cognitive function

Pharmacologically active concentrations of benzodiazepine receptor ligands have been found in serum and CSF from patients with hepatic encephalopathy ${ }^{1}$ and are correlated with its severity. ${ }^{2}$ Further evidence that benzodiazepine receptor ligands are involved in the pathogenesis of hepatic encephalopathy comes from clinical reports that the benzodiazepine receptor antagonist flumazenil produces significant improvements in neurological signs, ${ }^{34}$ from a double blind cross over trial of flumaze- nil in comatose patients, ${ }^{5}$ and from an uncontrolled trial of flumazenil in patients with latent hepatic encephalopathy. ${ }^{6}$

The pattern of psychomotor and memory impairments induced by benzodiazepines in healthy subjects is similar to that in hepatic encephalopathy. ${ }^{7}$ In a recent double blind placebo controlled study both alcoholic and non-alcoholic cirrhotic patients were significantly impaired in performance of psychomotor tasks. The episodic memory impairment of the alcoholic cirrhotic patients was reversed by flumazenil, which suggests that endogenous benzodiazepine receptor ligands contributed to this impairment. ${ }^{8}$

The purpose of the present study was to obtain measures of cognitive performance, circulating ligands for the neuronal benzodiazepine receptor, and benzodiazepine binding variables in platelets of alcoholic and nonalcoholic cirrhotic patients and matched controls. Our hypothesis was that the differences in cognitive performance between the groups would be related to differences in the concentrations of circulating ligands, to differences in benzodiazepine binding characteristics, or both, measured in platelets.

\section{Subjects}

Seventeen inpatients (nine alcoholic and eight non-alcoholic) from the Liver Unit, King's College Hospital with end stage cirrhosis (diagnosed by standard histological criteria) participated in the study, which had approval of local ethics committees; all subjects gave written informed consent. The non-alcoholic cirrhotic group had one of four liver diseases: primary biliary cirrhosis (two); primary sclerosing cholangitis (four); chronic active cirrhosis-hepatitis B (one); chronic active cirrhosis-hepatitis C (one). All patients included in the sample had grade I hepatic encephalopathy. We excluded from the sample patients with a history of neurological trauma or disease; chronic psychiatric disorders; current psychoactive drug intake; or benzodiazepine use within one month of the study.

International Classification of Diseases, 10th revision criteria were used to confirm a psychiatric diagnosis of alcohol misuse or dependence in the alcoholic patients as well as to exclude this in the other groups. The alcoholic patients had been drinking in excess of $80 \mathrm{~g} /$ day of alcohol for at least five years. They 
Characteristics of patients and control subjects (mean (SEM))

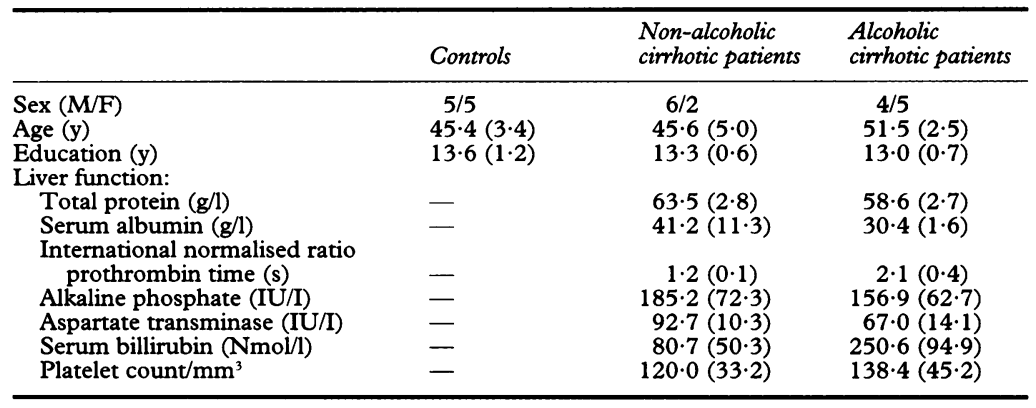

had ended their alcoholic beverage consumption at least 10 days before participation in this study. The mean duration of abstinence was 2.9 months (range: 11 days to six months). Alcohol drinking history was obtained during initial assessment and then checked with the data obtained in the patient's admission.

The controls were 10 normal volunteers matched to the patient groups for age and years of education (table). They were tested in the same conditions as the patients. None of the control subjects had a history of chronic medical illness, neurological injury, or disease and they were excluded if they had used benzodiazepines within six months of the study. They were asked to abstain from alcohol on the day before testing as a condition of entry to the study.

\section{Methods}

BLOOD SAMPLING

Venous blood samples $(40-50 \mathrm{ml})$ were obtained from patients and controls, collected into heparinised plastic tubes, and centrifuged at $180 \mathrm{~g}$ for 15 minutes and then at $190 \mathrm{~g}$ for five minutes, at $23^{\circ} \mathrm{C}$. Platelet rich plasma (supernatant) was removed and centrifuged at $1500 \mathrm{~g}$ for 15 minutes. The plasma was removed and stored at $-20^{\circ} \mathrm{C}$ and the remaining pellet, containing the platelets, was also frozen at $-20^{\circ} \mathrm{C}$.

\section{ESTIMATION OF ENDOGENOUS BENZODIAZEPINE} RECEPTOR LIGANDS

Endogenous ligands were extracted from plasma samples with chloroform. The level of benzodiazepine receptor ligands in each plasma sample was determined as described by Lund ${ }^{9}$ using $\left[{ }^{3} \mathrm{H}\right] \mathrm{Ro}-154513(2 \mathrm{nM})$, which labels $100 \%$ of the sites (both diazepam sensitive and diazepam insensitive sites) on the neuronal benzodiazepine receptor; (-) Bicuculline $(100 \mu \mathrm{M})(-)$ was added to block any action of GABA that remained after extraction. Non-specific binding was determined in the presence of $100 \mu \mathrm{M}$ flumazenil. A standard curve was constructed with known concentrations of flumazenil and the concentration of benzodiazepine receptor ligands estimated from this curve.

BENZODIAZEPINE BINDING IN PLATELETS

The number and affinity of peripheral benzodiazepine receptors were determined in the platelet enriched pellet, after one freeze-thaw and three washes to remove any circulating endogenous ligands. The receptor binding parameters were determined by Scatchard analysis, using eight concentrations of $\left[{ }^{3} \mathrm{H}\right]$ PK $11195(0 \cdot 3-20 \mathrm{nM})$ to achieve full saturation. ${ }^{10}$ Non-specific binding was determined in the presence of $1 \mu \mathrm{M}$ Ro 54864. The binding was quantified per $\mathrm{mg}$ of platelet protein. ${ }^{11}$

COGNITIVE AND PSYCHOMOTOR ASSESSMENTS

The cognitive tests were those previously shown to be sensitive to impairments found in alcoholic and non-alcoholic cirrhotic patients. ${ }^{8}$ The tests were brief and easily given at the bedside. They tapped memory (word recall), reaction times, focused attention (digit cancellation), and visual search/attention/mental flexibility, and motor speed (trails).

\section{Immediate and delayed recall}

Sixteen bisyllabic nouns were presented via a notebook computer and patients were asked to read the words aloud. Each word was displayed for two seconds and separated from the next word by one second. Patients were asked for spoken recall immediately after the last word was presented, and again after the delay filled by the tests below.

\section{Reaction times}

The patient was asked to press the space bar as quickly as possible after the appearance of a "flower" shape on the screen. Twenty four trials were given with random interstimulus intervals. The score is the median delay to pressing the bar.

\section{Digit cancellation}

The time taken to score out 40 number $4 \mathrm{~s}$ randomly interspersed among 400 numbers was recorded.

\section{Trails test}

This paper and pencil test has two parts, trails $\mathrm{A}$ and $\mathrm{B}$, each requiring the patient to complete a trail. ${ }^{12}$ Trails $A$ requires the patient to serially connect 25 encircled numbers with a continuous line; trails $B$ requires the alternate connection of 25 encircled numbers and letters (1-A-2-B-3-C- etc).

\section{Statistics}

The reaction time, digit cancellation, and peripheral benzodiazepine receptor binding data were analysed with one way analyses of variance (ANOVA) with the subject groups as the independent factor; the recall and trails data were analysed with ANOVAs with time of recall and trails task as the repeated measures and the subject groups as the independent factor. In all cases comparisons between individual groups was made after ANOVA with Duncan's tests (see fig 2). Spearman's correlation coefficients were calculated to determine the correlations between benzodiazepine receptor affinity and performance in the psychometric tasks. 


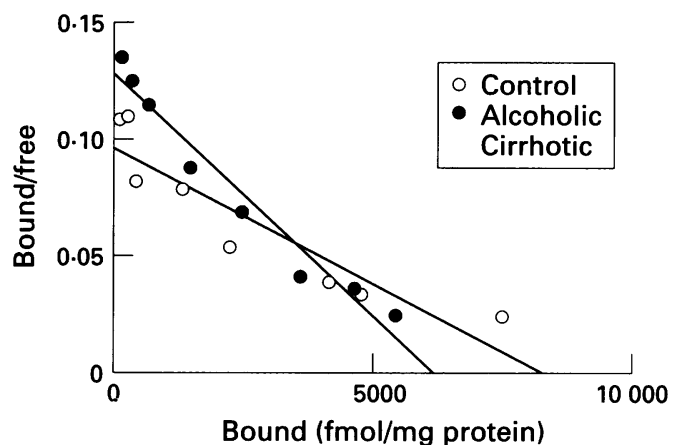

Figure 1 Scatchard plot of $\left.{ }^{3} H\right] P K 11195$ binding to frozen and thawed platelets from control subjects and alcoholic cirrhotic patients. $0.5 \mathrm{ml}$ samples were incubated for one hour at $4^{\circ} \mathrm{C}$ in eight different concentrations of [ $\mathrm{H}$ ]PK $11195(0 \cdot 3-20 \mathrm{nM})$ to achieve full saturation. Each point shows the mean at each ligand concentration for the control group $(O)$ and for the alcoholic cirrhotic group (O). Number of receptors is indicated by the intercept on the abscissa; Affinity is indicated by the slope.

\section{Results}

\section{LIVER FUNCTION}

There were no significant differences between the two patient groups in liver function (table). The higher INR and bilirubin in the alcoholic group just missed significance $(P=0 \cdot 10$ and $P=0.18$ respectively).

\section{ENDOGENOUS LIGANDS}

None of the control group had detectable ligands in the serum, but one of the non-alcoholic cirrhotic patients $(39 \cdot 1 \mathrm{nM}$ flumazenil equivalents) and three of the alcoholic patients $(8 \cdot 3,30 \cdot 4,13.4 \mathrm{nM})$ had measurable concentrations.

\section{BENZODIAZEPINE BINDING IN PLATELETS}

There was no detectable platelet binding in four alcoholic and four non-alcoholic cirrhotic patients and these patients also had platelet protein concentrations below the limits of our assay. There was no significant difference between the three groups in the number of receptors $(F(2,15)=1.6)$, but there was a significant difference in the receptor affinity $(F$ $(2,15)=4.4, P<0.05)$, with the alcoholic group having a higher affinity than the control group (fig 1); the affinity of the non-alcoholic group (6.2 (SEM 1.5)) did not differ from the controls $(5 \cdot 5(0.4))$.

\section{COGNITIVE AND PSYCHOMOTOR PERFORMANCE}

Immediate and delayed recall

Both groups of patients showed similar, pronounced impairments on both immediate and delayed word recall compared with controls $(F$ $(2,24)=8.3, P<0.005$; fig 2$)$. Forgetting (delayed compared with immediate recall) was similar in all three groups (group $\times$ time of recall interaction, $F(2,24)=0.03)$.

\section{Reaction times}

Both groups of patients showed slower reaction times than controls $(F(2,24)=4 \cdot 3$, $P<0.05$; fig 2).
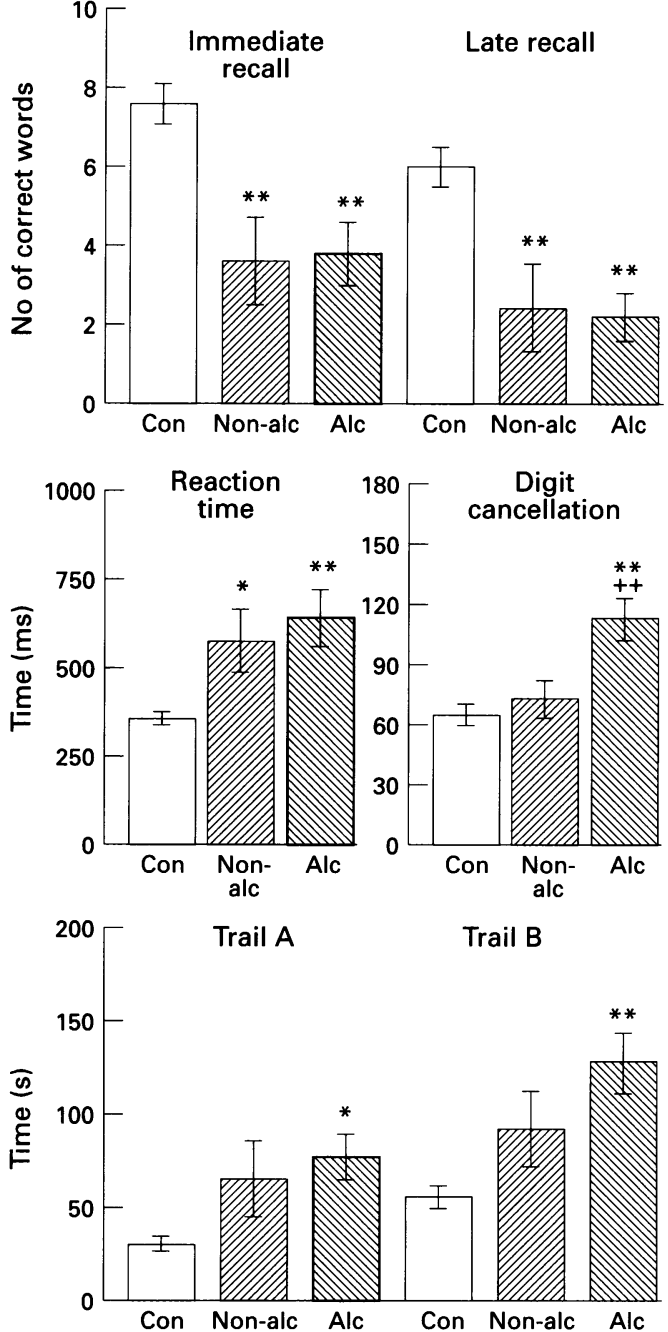

Figure 2 Mean (SEM) scores on the psychometric tests by control subjects, non-alcoholic and alcoholic cirrhotic patients. ${ }^{\star} P<0.05 ;{ }^{\star \star} p<0.01$ compared with controls; $++p<0.01$ compared with non-alcoholic cirrhotic group.

\section{Digit cancellation}

This task showed very clear differences between groups $(F(2,24)=9.0, \mathrm{P}<0.001)$. Whereas the alcoholic cirrhotic patients were severely impaired compared with controls, taking on average 50 seconds longer to complete this task, non-alcoholic patients were not impaired (fig 2).

\section{Trails test}

There was a significant difference between the performance of the groups on the trails tests $(F$ $(2,24)=5.3, P<0.01)$; alcoholic cirrhotic patients showed significant impairments compared with controls, and the non-alcoholic patients showed intermediate levels of performance (fig 2). Trails B took significantly longer than trails $A(P<0.00001)$ and the difference in performance of the two tasks was greater for the alcoholic patients $(P=0.06)$. Comparison with age graded normative data ${ }^{13}$ showed controls performing at normal levels, alcoholic cirrhotic patients performing below the 10th percentile on both trails $A$ and trails $B$, non-alcoholic cirrhotic patients performing below the 10th percentile on trails $A$ and just above the 25 th percentile on trails $B$. 
CORRELATION BETWEEN BENZODIAZEPINE FUNCTION AND PERFORMANCE

To determine whether the performance of the patients was related to the presence of endogenous ligands for the neuronal benzodiazepine receptor, the scores in the performance tests of patients with or without the presence of ligands were compared. There was no difference between the groups on reaction time or recall $(F(1,14)=<1.0$ in both cases). However, the groups did differ significantly in the time to complete the digit cancellation task (those with ligands taking 146.5 (SEM 16.0)s and those without $93(14 \cdot 7) \mathrm{s} ; F(1,14)=4.9$, $P<0.05)$ and there was a trend for greater impairment in the trails tasks for those with ligands $(P=0.07)$.

To determine whether there was any relation between the affinity of the benzodiazepine receptor and test performance, Spearman's correlation coefficients were calculated. These were significant only for reaction time and digit cancellation $(\rho=0.51$ in both cases, $\mathrm{P}<$ $0.05)$. Thus the higher the receptor affinity, the longer time taken to complete these tests.

\section{Discussion}

Although the two patient groups did not differ significantly in their liver function, this may have been due to the few patients studied. There was a clear trend for greater abnormalities in the alcoholic group and this increased impairment of liver function is likely to have resulted in the higher concentrations of endogenous ligands for the benzodiazepine receptor. These ligands could have originated from the food chain or gut fermentation products and impaired liver function would reduce their metabolic clearance. The cognitive differences between the two groups are likely to result from the CNS effects of alcohol adding to or interacting with the actions of benzodiazepine ligands on the CNS. In terms of cognitive performance, the alcoholic patients showed greater impairments than non-alcoholic patients and controls on the focused attention task (digit cancellation) and the trails tests. A relatively greater impairment in the alcoholic group on trails B compared with trails $\mathrm{A}$ emerged as both a nearly significant trend $(P=0.06)$ and in comparison with normative data, suggesting a level of executive dysfunction. Performance on the digit cancellation and trails tasks was related to the presence of endogenous ligands for the neuronal benzodiazepine receptor. Interestingly, it is also these tasks that are impaired in chronic benzodiazepine users. ${ }^{14}$ This raises the intriguing possibility that the impairments in attentional or executive function, particularly apparent in the alcoholic cirrhotic patients, might result from raised brain concentrations of benzodiazepine receptor ligands.

There was also a significantly enhanced affinity for the platelet benzodiazepine receptors in the alcoholic group. The receptor affinity correlated significantly with the times taken to complete both reaction time and digit cancellation tasks, suggesting a possible contribu- tion to motor slowing and impaired focused attention. A change in receptor affinity indicates that either some conformational change in the receptor has occurred, or that an endogenous ligand remained tightly attached to the receptor. Gavish and Fares ${ }^{15}$ found that the process of freezing, thawing, and washing membranes significantly enhanced the affinity of benzodiazepine receptors in the kidney and suggested that this process may have released a tightly bound endogenous ligand. Our binding assay used similar procedures and therefore one possibility is that higher concentrations of such a ligand were present in the alcoholic group. Peripheral benzodiazepine receptors differ in their pharmacological characteristics from the ones found on neurons ${ }^{16}$ and the present results suggest that alcoholic cirrhotic patients may have higher concentrations of circulating ligands, some of which are active at neuronal receptors and some of which are active at peripheral ones. Peripheral benzodiazepine receptors are found on various tissues, including the heart, kidney, and adrenals, where they control the rate limiting step in steroid synthesis. ${ }^{17}$ If changed affinity occurs in other peripheral tissues, this could contribute to a wide range of physiological symptoms. The peripheral type of benzodiazepine receptors also occur in the brain on glial cells, where they regulate the synthesis of neurosteroids, and an increased affinity has been reported in the brainstem of rats with experimental hepatic encephalopathy. ${ }^{18}$ However, it would seem unlikely that affinity changes will be found on all peripheral sites and, indeed, one study of patients with hepatic encephalopathy found no difference in the affinity of benzodiazepine receptors on lymphocytes, although the patients with hepatic encephalopathy had a reduced number of peripheral receptors compared with controls. ${ }^{19}$

In conclusion, our results have shown greater cognitive impairments in alcoholic, compared with non-alcoholic, patients with grade-I hepatic encephalopathy and suggest that these impairments may be linked to an increased incidence of endogenous ligands for the benzodiazepine receptor or to changes in receptor binding. Our conclusions have to be viewed with caution because of the small number of patients in which it was possible to measure platelet binding. This was because of the reduction in platelet concentration that is known to occur in hepatic encephalopathy ${ }^{20}$ and was indicated in our patients by unmeasurable plasma protein concentrations. It is thus likely to be a persisting problem in studying benzodiazepine binding in these patients. However, the small sample sizes are more likely to have obscured differences-for example, in the number of receptors-rather than produced false positives. The potential importance of these findings would certainly seem to warrant a more extensive study with a wider range of cognitive tests. All of our patients were assessed during a period of abstinence and there was no evidence that the extent of the biochemical changes could be linked to the duration of abstinence. Impaired liver function 
would affect the metabolism of endogenous benzodiazepine ligands and thus it is most likely that the results obtained reflect a combination of the effects of alcohol on the brain and liver disease.

These experiments were supported by a grant from the Special Trustees of Guy's Hospital.

1 Olasmaa M, Rothstein JD, Guidotti A, et al. Endogenous benzodiazepine receptor ligands in human and animal hepatic encephalopathy. $\mathcal{F}$ Neurochem 1990;55:2015-23.

2 Basile AS, Harrison PM, Hughes RD, et al. Relationship between plasma benzodiazepine receptor ligand concentrations and severity of hepatic encephalopathy. trations and severity of

3 Grimm G, Ferenci $P$, Katzenschlager $R$, et al. Improvement of hepatic encephalopathy treated with flumazenil. Lancet 1988;17:1392-4.

4 Bansky G, Meier PJ, Riederer E, Walser H, Ziegler WH, Schmid $M$. Effects of the benzodiazepine receptor antag onist flumazenil in hepatic encephalopathy in humans. Gastroenterology 1989;97:744-50.

5 Pomier-Layragues G, Giguere JF, Lavoie J, et al. Flumazenil in cirrhotic patients in hepatic coma: a randomized double-blind placebo-controlled crossover trial. Hepatology 1994;19:32-7.

6 Bruha R, Marecek Z, Brodanova M, Votruba M. Flumazenil as a diagnostic test for latent encephalopathy. $\mathcal{f}$ Hepatol 1994;20:844.

7 Curran HV. Benzodiazepines, memory and mood: review. Psychopharmacology 1991;105:1-8.

8 Kapczinski F, Sherman D, Williams R, Lader M, Curran $V$. Differential effects of flumazenil in alcoholics and nonalcoholic cirrhotic patients. Psychopharmacology 1995;120:220-6.
9 Lund J. Radioreceptor assay for benzodiazepines in biological fluids using a new dry and stable receptor preparacal fluids using a new dry and stable receptor

10 Gavish M, Weizman A, Karp L, Tyano S, Tanne Z. Decreased peripheral benzodiazepine binding sites in pecreased peripheral benzodiazepine binding sites in platelets of neuroleptic-tr

11 Lowry OH, Rosebrough NJ, Farr AL, Randall RJ. Protein measurement with the Folin phenol reagent. $f$ Biol Chem 1951;193:265-75.

12 Reitan RM. Validity of the trail making test as an indication of organic brain damage. Percept Mot Skills 1958;8: $271-6$.

13 Spreen A and Strauss S. A compendium of neuropsychological tests: administration norms and commentary, Oxford: Oxford University Press, 1991.

14 Golombok S, Moodley P, Lader M. Cognitive impairment in long-term benzodiazepine users. Psychol Med 1988; 18:365-74.

15 Gavish $M$, Fares $F$. The effect of freezing and thawing or of detergent treatment on peripheral benzodiazepine binding: the possible existence of an endogenous ligand. Eur $\mathcal{F}$ ing: the possible existence of

16 Gavish M, Katz Y, Bar-Ami S, Weizman R. Biochemical, physiological, and pathological aspects of the peripheral benzodiazepine receptor. F Neurochem 1992;58:1589-601.

17 Krueger KE, Papadopoulos V. Peripheral type benzodiazepine receptors modulate translocation of cholesterol from outer to inner mitochondrial membranes in adrenocortical cells. F Biol Chem 1990;265:15015-21.

18 Zhang ZM, Qui FZ, Chen XP. Experimental studies on peripheral-type benzodiazepine receptors in brain in rats with hepatic encephalopathy. Chinese foumal of Surgery 1994;32:37-40

19 Ferrero P, Rocca P, Gualerzi A, et al. A study of 3H-PK 11195 binding to "peripheral-type" benzodiazepine receptors on human lymphocytes. ₹ Neurol Sci 1991; 102:209-19.

20 Stein SF, Harper LA. Kinetic and functional studies of platelets, fibrinogen, and plasminogen in patients with hepatic cirrhosis. F Lab Clin Med 1982;99:21 7-30. 